4 Mota NB, Vasconcelos NA, Lemos N, Pieretti AC, Kinouchi O, Cecchi GA, et al. Speech graphs provide a quantitative measure of thought disorder in psychosis. PLOS One 2012; 7: e34928.

Philip Corlett, Sarah Fineberg, Yale University Department of Psychiatry, Connecticut Mental Health Center, New Haven, CT, USA. Email: philip.corlett@yale.edu

doi: 10.1192/bjp.205.5.409a

\section{BDNF and proBDNF as biomarkers for bipolar disorder}

I read with great interest the recent article by Li et al, describing plasma levels of brain-derived neurotrophic factor (BDNF) in patients with bipolar disorder in their first depressive episode. ${ }^{1}$ A total of 203 patients with a first major depressive episode, as well as 167 healthy controls, were enrolled. After 3 years of bi-annual follow-up, 164 patients with a major depressive episode completed, and of these, 21 patients were diagnosed as having bipolar disorder and 143 patients were diagnosed as having major depressive disorder. At baseline, patients with bipolar disorder and depression showed significantly lower BDNF mRNA levels $(P<0.001$ and $P=0.02$, respectively) and plasma BDNF levels ( $P=0.002$ and $P=0.01$, respectively) compared with healthy controls. Interestingly, plasma BDNF levels in patients with bipolar disorder were lower than those in patients with depression.

This study suggests that the model for predicting bipolar disorder during a first depressive episode is a combination of BDNF mRNA with plasma BDNF levels. ${ }^{1}$ BDNF (mature BDNF) is a $13 \mathrm{kDa}$ polypeptide, which is initially synthesised as a precursor protein, preproBDNF, in the endoplasmic reticulum. Following cleavage of the signal peptide, proBDNF $(\sim 32 \mathrm{kDa})$ is converted to mature BDNF by extracellular proteases. It was initially thought that only secreted, mature BDNF was biologically active, and that proBDNF, localised intracellularly, served as an inactive precursor. However, accumulating evidence shows that both proBDNF and mature BDNF are active, eliciting opposing effects via the p75NTR and TrkB receptors, respectively, and that both forms play important roles in several physiological functions. $^{2}$

The enzyme-linked immunosorbent assay (ELISA) kits (R\&D Systems) used by Li et al recognise both proBDNF (precursor of BDNF) and mature BDNF, because of the limited specificity of the BDNF antibody. ${ }^{3}$ Using newly available human proBDNF and mature BDNF ELISA kits, which differentiate between the BDNF forms, we have reported high levels of both proBDNF and mature BDNF in human serum. ${ }^{3}$ We reported that serum levels of mature BDNF, but not proBDNF, in patients with major depressive disorder were significantly lower than those in healthy controls. ${ }^{4}$ And we recently found that serum levels of mature $\mathrm{BDNF}$ and the ratio of mature BDNF to proBDNF in moodstabilised patients with bipolar disorder were significantly higher than in healthy controls. ${ }^{4}$ Interestingly, serum levels of proBDNF in mood-stabilised patients with bipolar disorder were significantly lower than those in healthy controls. ${ }^{5}$ These findings were confirmed in two independent cohorts (Sahlgrenska set and Karolinska set in Sweden). ${ }^{5}$ Considering the high levels of both proBDNF and mature BDNF in human serum, and their putative opposing functions, it would be clinically and scientifically interesting to measure the individual serum levels of proBDNF and mature BDNF in this cohort study.

1 Li Z, Zhang C, Fan J, Yuan C, Huang J, Chen J, et al. Brain-derived neurotrophic factor levels and bipolar disorder in patients in their first depressive episode: 3-year prospective longitudinal study. Br J Psychiatry 2014; 205: 29-35.

2 Hashimoto K. Brain-derived neurotrophic factor as a biomarker for mood disorders: an historical overview and future directions. Psychiatry Clin Neurosci 2010; 64: 341-57.
3 Yoshida T, Ishikawa M, Iyo M, Hashimoto K. Serum levels of mature brainderived neurotrophic factor and its precursor proBDNF in healthy subjects. Open Clin Chem J 2012; 5: 7-12.

4 Yoshida T, Ishikawa M, Niitsu T, Nakazato M, Watanabe H, Shiraishi T, et al. Decreased serum levels of mature brain-derived neurotrophic factor (BDNF), but not its precursor proBDNF, in patients with major depressive disorder. PLOS One 2012; 7: e42676.

5 Södersten $\mathrm{K}$, Pílsson $\mathrm{E}$, Ishima $\mathrm{T}$, Funa $\mathrm{K}$, Landén $\mathrm{M}$, Hashimoto $\mathrm{K}$, et al. Abnormality in serum levels of mature brain-derived neurotrophic factor (BDNF) and its precursor proBDNF in mood-stabilized patients with bipolar disorder: a study of two independent cohorts. J Affect Dis 2014; 160: 1-9.

Kenji Hashimoto, Professor, Chiba University Center for Forensic Mental Health. Email: hashimoto@faculty.chiba-u.jp

doi: 10.1192/bjp.205.5.410

\section{Declaration of interest}

K.H. is a holder of the patents 'Diagnostic and examination method for eating disorder' (US 7,754,434 B2) and 'Diagnostic agent for ischemic heart disease risk group' (US 2013/ 0310321A1), which pertain to the measurement of BDNF as a biomarker. In addition, He has served as a scientific consultant to Astellas and Taisho and he has received research support from Abbvie, Dainippon Sumitomo, Otsuka and Taisho.

Authors' reply: While we agree with Professor Hashimoto's comments regarding the predictive role of mature brain-derived neurotrophic factor (mBDNF) and its precursor, proBDNF, in bipolar disorder, several points merit further discussion.

First, we presented preliminary data describing a potential role for BDNF as a biomarker for predicting bipolar disorder in major depressive disorder, although we detected the serum BDNF level using commercial kits that do not differentiate between mBDNF and proBDNF. When we reviewed the literature regarding mBDNF and proBDNF in bipolar disorder and major depressive disorder, we noticed that lower serum levels of mBDNF and higher serum levels of proBDNF were found among patients with major depressive disorder. ${ }^{1,2}$ Södersten et al also reported that higher serum levels of mBDNF and lower proBDNF were observed among patients with bipolar disorder. ${ }^{3}$ These disparate results suggest that levels of mBDNF and proBDNF, as well as the ratio of mBDNF to proBDNF, might be sensitive enough to help differentiate bipolar disorder from major depressive disorder.

Second, our previous studies indicated that BDNF probably has some sex-specific characteristics. Tang et al ${ }^{4}$ reported that the ratio of $\mathrm{mBDNF}$ to proBDNF differs in a sex-specific manner in zebra finches. These findings suggest that $\mathrm{mBDNF}$ and proBDNF are different in males and females and should be further investigated.

Third, the findings of one of our previous studies implied that genetic interactions between genes encoding BDNF and its receptor enhance the risk of treatment-resistant depression. ${ }^{5}$ Recent studies have found that $\mathrm{mBDNF}$ and proBDNF elicit biological effects via interaction with their respective receptors, p75NTR and TrkB. Accordingly, we concluded that evaluations of mBDNF and proBDNF should also consider their receptors. On the whole, we appreciate Professor Hashimoto's insightful comments in directing our future work.

1 Yoshida T, Ishikawa M, Niitsu T, Nakazato M, Watanabe $H$, Shiraishi $T$, et al. Decreased serum levels of mature brain-derived neurotrophic factor (BDNF), but not its precursor proBDNF, in patients with major depressive disorder. Plos One 2012; 7: e42676.

2 Zhou L, Xiong J, Lim Y, Ruan Y, Huang C, Zhu Y, et al. Upregulation of blood proBDNF and its receptors in major depression. J Affect Disord 2013; 150: 776-84.

3 Södersten $\mathrm{K}$, Palsson $\mathrm{E}$, Ishima T, Funa $\mathrm{K}$, Landen $\mathrm{M}$, Hashimoto $\mathrm{K}$, et al. Abnormality in serum levels of mature brain-derived neurotrophic factor (BDNF) 